\title{
Non-Linear Directive Contrast Filter for Mammogram Images to Enhance Pleomorphic Calcification
}

\author{
Ramya A. \\ Research Scholar \\ Dept.of CSE, \\ Manonmaniam Sundaranar \\ University
}

\author{
V. Murugan, PhD \\ Assistant Professor \\ Dept.of CSE, \\ Manonmaniam Sundaranar \\ University
}

\author{
D. Murugan, $\mathrm{PhD}$ \\ Professor \\ Dept.of CSE, \\ Manonmaniam Sundaranar \\ University
}

\begin{abstract}
Breast cancer is now wide spread among the women at the age of 35 and above. Initial stage of breast cancer is calcification. Mammography is the precise type of imaging source for breast cancer and calcification deposit in breast, which are usually low contrast mammogram images. This paper deals with the analysis of suspicious or intermediate coarse type of calcification in the breast. Pleomorphic is a kind of the suspicious calcification deposition in the breast, which may lead to cancerous stage, if not examined earlier. Enhancement is one of the pre-processing stage used to improvise the contrast, interpretation and perception of the image, so that calcium deposited areas in the mammogram images can be viewed evidently. Histogram equalization is most significant method for improving the visual perception of medical images. In this paper, before now proposed system of Histogram equalization technique such as RMSHE (Recursive Mean-Separate Histogram equalization), AMHE (Adaptively Modified Histogram Equalization), BPDFHE (Brightness Preserving Dynamic Histogram Equalization) were studied under experimental analysis and compared with our proposed technique such as Non-Linear Directive (NLDC) filter to progress the low-level intensity of an image. Comparison of this technique with the proposed filter is necessary for deciding appropriate algorithms for enhancing the medical images. Quality evaluation factors for image enhancement like PSNR (Peak Signal to Noise Ratio), MSE (Mean Squared Error), Michelson Contrast and AMBE (Absolute Mean Brightness Error) were also analyzed for the existing and proposed technique. The proposed technique yields a better outcome than the other compared technique..
\end{abstract}

\section{Keywords}

Non-Linear, Image Enhancement, Pleomorphic Calcification, Mammogram, Histogram Equalization, Contrast, Intensity.

\section{INTRODUCTION}

The field of medical imaging advancing rapidly with the development of new devices and expertise. Breast cancer is the most commonly observed cancer in women both in the developing and the developed countries of the world. Cancer usually refers to the unrestrained growth of a group of cells in a certain location of the body. Generally breast calcification starts with calcium deposit. In some cases it may not harmful, but later examining may cause serious issues. The calcifications seen on a mammogram images as small white spots. They usually deposited anywhere in the breast tissue which does not cause any kind of pain. It is very common and habitually develops at the age above 35 in women mostly in the western countries. Some calcification are not risk causing agent, they are usually called benign (not cancerous type). But some calcifications can rarely be an early sign of cancer.

In some types of breast calcifications, the calcium can widen in the blood vessels of the breast. Radiologist carefully examine over their three important features they are: size, shape and pattern. Breast calcification generally divided into micro and macro calcification. Calcification is small spots of calcium substance deposit in tissues or blood vessels in the breast. Generally the shape of calcifications is round, popcorn like, large rod like or granules. Pattern of the calcification commonly scattered, linear line or group cluster. Benign case are produced from screening exams in which something suspicious (intermediate concern) was found, but not determined to be malignant. Determining the malignant usually done by the pathologist.

According to the international agency for research on cancer, around 79,000 women's per year affected by breast cancer in India [2]. The National Cancer Institute estimates that one of the eight women in the United States breast cancer will develop at some point during her lifetime [4].

Digital mammogram becomes the most effective technique for early breast cancer uncovering modality and handing out these images requires high computational capabilities [5]. Mammography is a low dose $\mathrm{x}$-ray procedure for the visualization of internal structure of breast. Mammography has been proven the most reliable method and it is the key screening tool for the early detection of breast cancer. Mammography is highly accurate, but like most medical tests, it is not perfect. On average, mammography will detect about $80-90 \%$ of the breast cancers in women without symptoms [1].

Image enhancement process inherits a collection of techniques which helps to progress the image appearance better to scrutinize by humans. This process generally classifies into two categories they are spatial and frequency domain techniques. Former technique directly deals with the pixel of a digital image. Later methods deals with Fourier transform approach. This work intensively in the spatial process of histogram equalization, which is most recurrently, used technique in which distribution of gray scale values will be consistent. In general contrast enhancement is divided into two distinct category namely direct and indirect contrast enhancement. Indirect contrast enhancement involves modifying of the histogram [14].

For evaluating the risk of suspicious calcification such as pleomorphic calcification, the dataset from DDSM (Digital Database for Screening Mammogram) is employed and analyzed for interpretation of pleomorphic calcification. It is a 
USF (University of South Florida) database available publically.

\section{RELATED WORK}

Mammography plays an essential role in early detection of breast cancers because it can show changes in the breast up to two years before a patient or physician can feel them. Early detection of suspicious calcification which leads to cancer is also curable. However the initial indication of breast cancer is formation of a new lump or mass in the breast. A lump or mass that is painless, hard, and has uneven edges is more possible to be cancer. But sometimes cancerous lump or mass can be tender, soft, and rounded. The pre-processing is based on an enhancement and segmentation steps of the mammogram. Image enhancement is the process of manipulating an image so that the result is more suitable than the origin for a specific application. The word specific is important because it establishes at the outset that enhancement techniques are problem oriented. In addition, removing noise is also important during the enhancement technique [3]. The above diagrammatic representation depicts the calcification morphology in the breast. Benign is not usually a cancerous risk, but intermediate or suspicious calcification may or may not be cancerous. Generally earlier detection of the suspicious calcification may not lead to cancer tissue or mass formation. Malignant distribution is definite cancerous mass that should be removed. In this paper, Pleomorphic calcification which has malignant feature is analyzed by improving the visual perception of the mammogram image by using various enhancement technique. Pleomorphic calcification is the initial stage of the cancerous but not purely malignant, it may also distribute under the suspicious category. Vascular calcification is linear parallel tracks that are generally clearly allied with blood vessels. Popcorn-like calcification is produced by involution fibroadenoma.

When the calcifications in a fibroadenoma are small and numerous, they may resemble malignant-type calcifications and need a biopsy. Rod-like and Plasma cell mastitis are formed within the ducts. These benign calcifications form continuous rods that may occasionally be branching.

Eggshell or Rim Calcification is very thin benign calcifications that appear as calcium is deposited on the surface of a sphere. Fine Pleomorphic calcifications vary in size and shapes.

There is a $25-40 \%$ risk of malignancy with this type of suspicious calcification. Fig 2 (d) shows calcification of pleomorphic calcification in a cluster as classified as BIRADS 4. The most conspicuous calcifications however are the fine pleomorphic calcifications and it has segmental distribution [10].

Image processing operations involving enhancement makes use of oriented filters, same filter is applied over again by rotating it at different orientations under adaptive control. One can remove noise and enhance oriented structures by angularly adaptive filtering [11]. But by applying the same filter repeatedly over the same image, it may sometimes over brighten the very small feature, so there may be chances of missing some features. Traditional enhancement methods like Weiner filter is suitable for denoising the image is good for increase the contrast of the image [12]. Excluding the contrast, while taking the histogram for these images the gray level values are not equally distributed.

Computer image processing techniques will be applied to enhance images and this is followed by segmentation of the region of interest (ROI). Subsequently, the textural features will be extracted from the ROI. The texture features will be used to classify the ROIs as either masses or non-masses [13] While segmenting the cancerous area based on the textural features, some area of the scattered calcification may be missed.

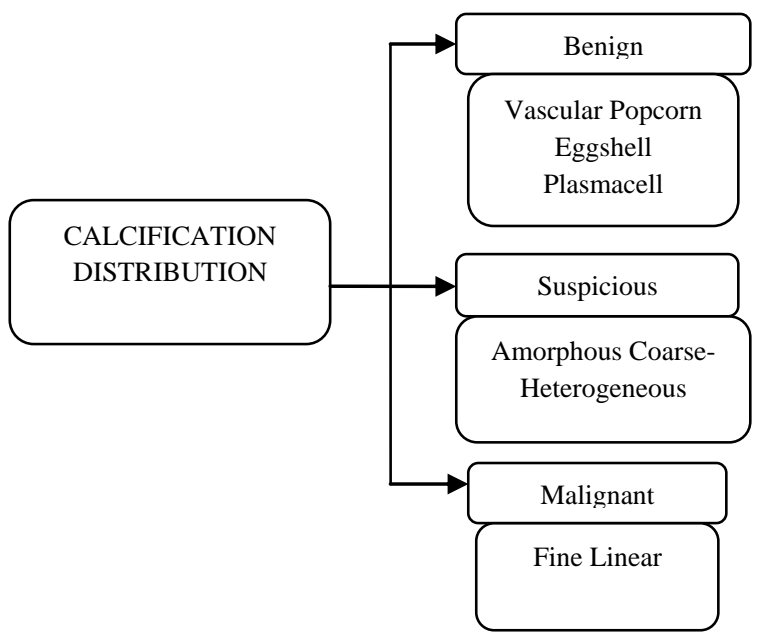

Fig.1. Distribution of the Calcification

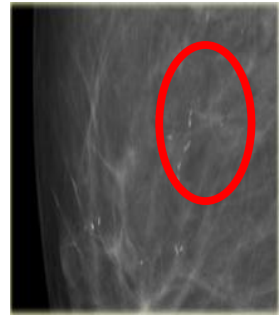

Fig 2. (a)

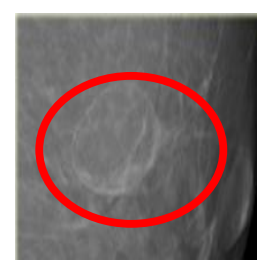

(d)

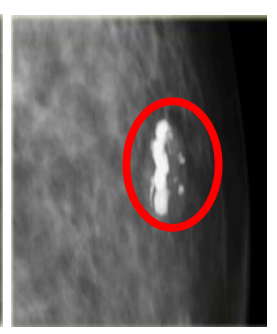

(b)

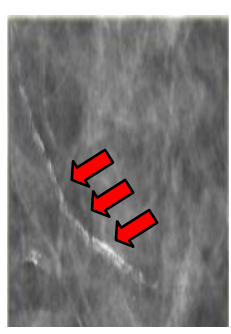

(c)

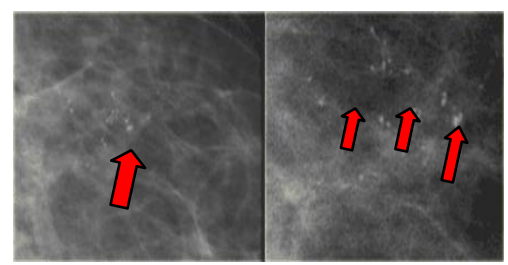

(e)(i)

(e)(ii)

(a)Tiny rod like (b) popcorn like (c) Vascular (d)Eggshell (e)(i) Pleomorphic calcification (e)(ii) zoomed image of Pleomorphic calcification

\section{HISTOGRAM EQUALIZATION TECHNIQUE}

Image Enhancement is the process of adjusting digital image to improve the quality of the image. It is a subjective process. Histogram equalization (HE) is a very common method for enhancing the contrast of an image [6]. Various histogram equalization techniques have been studied and carried out this work. They are:

- Recursive Mean-Separate Histogram Equalization (RMSHE)

- Adaptively Modified Histogram Equalization (AMHE)

- $\quad$ Brightness Preserving Dynamic Fuzzy Histogram Equalization (BPDFHE) 


\subsection{Recursive Mean-Separate Histogram Equalization (RMSHE)}

The generalization of Bi-Histogram Equalization (BBHE) referred to as Recursive Mean Separate Histogram Equalization (RMSHE) to provide not only better but also scalable brightness preservation. BBHE separates the input image's histogram into two, based on its mean, but RMSHE perform the separation recursively. When the number of mean separations increases recursively, the mean brightness of an input image will also increase. Therefore, the scalability in this algorithm is the most desirable property to allow adjustment of the level of brightness preservation base on individual image's requirement [7]. Recursive Mean-Separate Histogram Equalization (RMSHE) gives the better result or enhancing the medical image. RMSHE techniques were implemented and its performed metrics have also been analyzed. The brightness of the resultant image is better preserved as $\mathrm{r}$ increases (recursion level). As summarized in the above mentioned RSIHE technique, finding of $r$ value is difficult and its value should be consistence to find the better enhancement result.

\subsection{Adaptively Modified Histogram Equalization (AMHE)}

The contrast enhancement method called adaptively modified histogram equalization (AMHE) is an expansion of the typical histogram equalization method. To prevent any significant change of gray levels between the original image and histogram equalized image, the AMHE scales the magnitudes of the probability density function of the original image before equalization. AMHE techniques, scales the magnitudes of the probability density function (PDF) of the original image before equalization. AMHE controls the rate of contrast enhancement that can be adaptively resolute based on the mean brightness an image. AMHE can prevent a significant change in gray level by scaling the PDF [8]. The gray levels are stretched in proportion to those probabilities in AMHE and thus the contrast of the regions in the image composed of those gray levels is more enhanced. The experimental results indicate that AMHE not only improves the contrast effectively, but also keeps the tone of the original image to some extents. This method enhances the intensity and contrast of an image gradually so that the darker areas of an image will became even darker.

\subsection{Brightness Preserving Dynamic Fuzzy Histogram Equalization (BPDFHE)}

Brightness Preserving Dynamic Fuzzy Histogram Equalization (BPDFHE) is the novel modification of the brightness preserving dynamic histogram equalization (BPDHE) technique to improve its brightness preserving and contrast enhancement abilities while reducing its computational complexity. BPDFHE uses fuzzy statistics of digital images for their representation and processing. Representation and processing of images in the fuzzy domain enable the technique to handle the imprecision of gray level values in a better way [9].

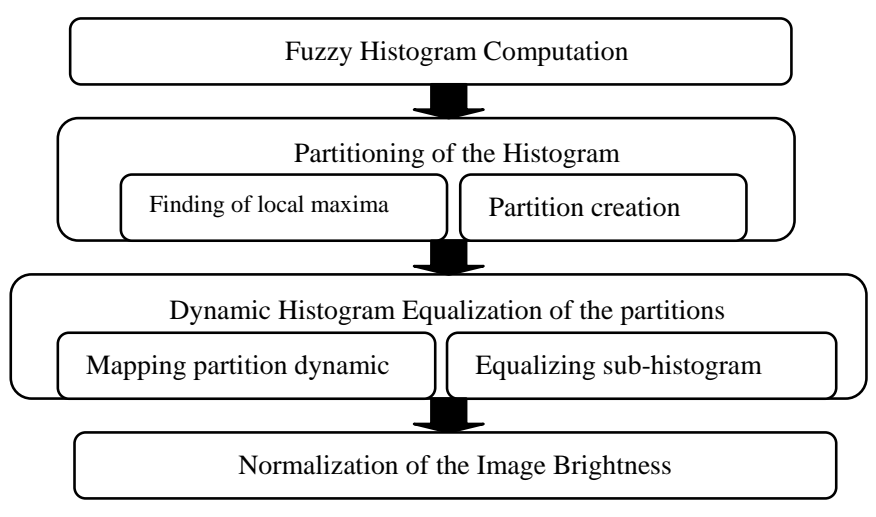

Fig 3. Steps involved in BPDFHE

The efficient change in mean value has certain image. The overall ratio of the resultant image is better than AMHE and RMSHE but it has the limitation over its computing time.

\section{PROPOSED METHODOLOGY}

The proposed framework for image enhancement uses the Non-Linear Directive Contrast Filter (NLDC). The nonlinear strategy improvises the minute characteristics in every range without any blurring at the edges of the ROI (Region of Interest) feature in the mammogram images.

This methodology initially uses the histogram gray level distribution of ROI for finding the minimum and the maximum value from it. Imin, Imax is the minimum and the maximum intensity values obtained from the histogram. Partitioning of the histogram is done based upon the mean value obtained from the gray level distribution by the equation (1).

$$
\left[\operatorname{Imin}, i_{0}\right],\left[i_{0_{+1}}, i_{1}\right],\left[i_{0_{+2}}, i_{2}\right], \ldots \ldots . .\left[i_{n+1}, I \max \right]
$$

The imprecision in the gray level is reduced by partitioning the histogram. After applying the histogram, it is then subjected to the luminance factor to distinguish the foreground and background intensity to make a clear of the exaggerated portion in an image. This is necessary because pleomorphic calcification in mammogram image usually linearly clustered area, so to improvise that foreground part from the dense tissue luminance factor is applied to mammogram image.

$$
C_{(i, j)}=\frac{L_{1}-L_{i}}{L_{i}}=\frac{L_{a}}{L_{i}}
$$

Where $\mathrm{L} 1$ is the foreground luminance and $\mathrm{Li}$ is the luminance of the background an image. Generally this process enhance the high frequency elements in an image to an elevated scope, which usually makes all the details and noises in an image will get enhance to a greater extend. The outcome of luminance factor is subjected to non-linear directive filter to normalize the noise, high frequency coefficient and also the gray level distribution.

$$
N L D=\frac{\left[C_{(i, j)}-\operatorname{Imin}(x)\right]}{[\operatorname{Imax}(x)-\operatorname{Imin}(x)]}
$$

Where $C_{(i, j)}$ represent the input image, min and max are the minimum and maximum value obtained from gray level distribution. This filter works with the spatial domain filter. This filter has no gradient misrepresentation near the edges of an image and also helps to hold back the noise near the edges. 
Pseudo code for performing the Non-Linear Directive Contrast:

Step 1: Read an image

Step 2: Perform the histogram gray level distribution function

Step 3: Partition the histogram with maximum and minimum mean value

Step 4: Use the luminance factor for the background and foreground features to enhance

Step 5: Carry out non-linear equation to the subjected luminance factor image.

Step 6: Display the enhanced image.

After processing of these algorithm steps, the enhanced output image is produced. The described filter has been tested on nearly 150 mammographic images. The sample of experimental image is given $\mathrm{n}$ figure 5 . The corresponding performance factor such as Peak Signal-to-Noise Ratio (PSNR), Mean Squared Error (MSE), Absolute Mean Brightness Error (AMBE), Michelson Contrast has also been analyzed for NLDC filter and other compared method. The result obtained from the proposed filter produced better than other technique.

\section{EXPERIMENTAL OUTCOME}

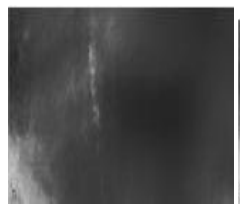

Fig 5. (a)

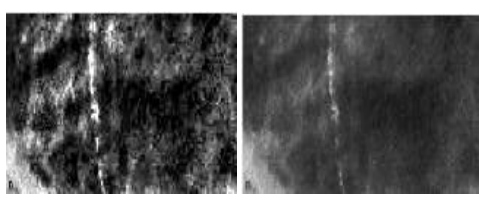

(a) input pleomorphic calcification of mammogram image (b) RMSHE (c) AMHE (d) BPDFHE (e) Proposed filter

The above figures represent the experimental outcome of pleomorphic calcification of mammogram image. We have worked with 150 image dataset for each technique described in this paper. Instead of processing with the entire image area, ROI is taken from the mammogram image and the various methodology of luminance and contrast factor is applied. Initially in the ROI input image some of the calcification deposited areas were missing. After processing the input mammogram images with RMSHE, AMHE, BPDHE and NLDC filter, the later methods yields the clear perception of the calcification formation than the other three methods. In BPDHE techniques, calcification were clearly detected but other than the calcification areas were also been increased with their gray level values. So there may be misconception in detection of calcification in other area also. In RMSHE and AMHE techniques, some of the actual area of calcification is smoothed out but in our proposed filter the area of calcification is clearly shown and edges are preserved.

\subsection{Experimental Evaluation}

The following result shown in the below tables are noise estimated parameters for enhancement outcome. The PSNR (Peak Signal to Noise Ratio), MSE (Mean Squared Error), Michelson Contrast, AMBE (Absolute Mean Brightness
Error) value the few existing techniques and compared with the proposed method for Pleomorphic calcification images. MSE is given by the formula.

$$
M S E=(1 / M N) \sum_{m=0}^{M-1} \sum_{n=0}^{N-1} e(m, n)
$$

PSNR is the standard evaluation for determining the noise level in an image. PSNR is measured in decibels $(\mathrm{dB})$ and is given by.

$$
P S N R=10 \log \left(S^{\wedge} 2 / M S E\right)
$$

Michelson contrast is normally used for estimating the intensity levels of an image. It usually deals with the both bright and dark features in an image.

$$
\text { MichelsonContrast }=\frac{l_{\max }-l_{\min }}{l_{\max }+l_{\min }}
$$

Where $I_{\max } \& I_{\min }$ are maximum and minimum intensity value of an image. This value should be 1 which proves that total range of image is maintained constant after preprocessing.

AMBE is the difference between the brightness of the original image and output image. The formula for AMBE is.

$$
A M B E=|E(x)-E(y)|
$$

Where $E(x)$ is average intensity of input image $E(y)$ is average intensity of enhanced image.

Table 1. PSNR evaluation for mammogram images

\begin{tabular}{|l|l|l|l|l|}
\hline Image & RMSHE & AMHE & BPDFHE & NLDC \\
\hline Imgdb01 & 20.503 & 29.382 & 35.007 & 52.185 \\
\hline Imgdb05 & 20.128 & 30.896 & 40.598 & 50.254 \\
\hline Imgdb10 & 20.968 & 30.158 & 47.258 & 48.198 \\
\hline Imgdb15 & 17.457 & 29.759 & 39.540 & 51.892 \\
\hline Imgdb20 & 19.782 & 29.452 & 42.658 & 50.125 \\
\hline Imgdb25 & 17.897 & 28.077 & 40.157 & 50.017 \\
\hline Imgdb30 & 20.018 & 29.869 & 40.254 & 49.875 \\
& & & & \\
\hline
\end{tabular}

From 150 test images, sample of 30 pleomorphic affected mammogram images is tabled. The evaluation yields from the above table shows the PSNR estimation for RMSHE, AMHE, BPDFHE and proposed method. The proposed technique gives better normalized value for the test dataset.

Table 2. MSE evaluation for mammogram images

\begin{tabular}{|l|l|l|l|l|}
\hline Images & RMSHE & AMHE & BPDFHE & NLDC \\
\hline Imgdb01 & 2.452 & 5.511 & 1.591 & 0.396 \\
& & & & \\
\hline Imgdb05 & 2.597 & 3.715 & 0.758 & 0.157 \\
& & & & \\
\hline
\end{tabular}




\begin{tabular}{|l|l|l|l|l|}
\hline Imgdb10 & 4.177 & 4.187 & 1.417 & 0.564 \\
\hline Imgdb15 & 2.579 & 4.851 & 1.971 & 1.237 \\
\hline Imgdb20 & 2.781 & 4.007 & 1.608 & 0.627 \\
\hline Imgdb25 & 2.007 & 5.015 & 2.187 & 1.287 \\
& & & & \\
\hline Imgdb30 & 2.568 & 4.892 & 1.125 & 0.458 \\
\hline
\end{tabular}

MSE is the Mean Squared Error measure for analyzing the noise rate in an image. Usually this error estimation should be minimum, which means image is not much affected with mean error of noise. The outcome result for the proposed method seems to be better result compared with the other HE techniques.

Table 3. Michelson Contrast evaluation for mammogram images

\begin{tabular}{|l|l|l|l|l|}
\hline Images & RMSHE & AMHE & BPDFHE & NLDC \\
\hline Imgdb01 & 0.908 & 0.808 & 0.991 & 1 \\
\hline Imgdb05 & 0.862 & 0.908 & 0.897 & 1 \\
\hline Imgdb10 & 0.729 & 0.936 & 0.995 & 0.978 \\
\hline Imgdb15 & 0.877 & 0.821 & 0.984 & 1 \\
\hline Imgdb20 & 0.891 & 0.878 & 0.781 & 0.988 \\
\hline Imgdb25 & 0.887 & 0.984 & 0.845 & 1 \\
\hline Imgdb30 & 0.997 & 0.842 & 0.935 & 1 \\
\hline
\end{tabular}

Michelson Contrast is generally called as Weber fraction. It indicates that for a large background the small features should posses the constant value. The projected method gives a constant value for the 20 image dataset around $90 \%$.

Table 4. AMBE parameter for mammogram images

\begin{tabular}{|l|l|l|l|l|}
\hline Images & RMSHE & AMHE & BPDFHE & NLDC \\
\hline Imgdb01 & 7.2541 & 4.1280 & 0.1852 & 0.0430 \\
\hline Imgdb05 & 10.6260 & 3.4513 & 0.1687 & 0.0861 \\
\hline Imgdb10 & 9.7254 & 3.9247 & 0.1485 & 0.0587 \\
\hline Imgdb15 & 6.1864 & 3.7197 & 0.0962 & 0.0497 \\
\hline Imgdb20 & 9.7822 & 4.1243 & 0.0874 & 0.1017 \\
\hline Imgdb25 & 9.2471 & 3.1478 & 0.0975 & 0.0457 \\
\hline Imgdb30 & 8.2547 & 3.3247 & 0.1526 & 0.0185 \\
\hline
\end{tabular}

The value of the AMBE increases it means that there is wide difference for the input and output image which may sometimes over brighten an image. Here in the above inferences proposed method yields a little difference from original image to output image, so it clearly seems that gray level values are equally distributed and mapped.

\section{CONCLUSION}

This paper addressed the issues of enhancing the low contrast mammogram images using Non-linear Directive Contrast (NLDC) filter which directly works with the intensity and made clear visual perception of breast calcification and diagnosis. We have also computed the luminance factor before applying the directive filter. The ROI of calcification part is well enhanced with our proposed filter. The framework of this work is compared with three different histogram equalization methods such as BPDFHE (Brightness preserving dynamic fuzzy histogram equalization), RMSHE (Recursive Mean-Separate Histogram Equalization) and AMHE (Adaptively modified histogram equalization). By observing the evaluation outcomes and qualitative factors for existing and proposed method, the projected technique yields a much better outcome. The future work can be carried out with the earlier segmentation of pleomorphic calcification which will be helpful for detecting the actual and concrete area of calcification accurately. This work can be also carried out with the continuation, by analyzing the texture of the calcification area after applying Non-Linear Directive Contrast filter.

\section{ACKNOWLEDGMENTS}

Our sincere thanks to the experts who have contributed towards development of the template.

\section{REFERENCES}

[1] Ramani, R., N. Suthanthira Vanitha, and S. Valarmathy. "The pre-processing techniques for breast cancer detection in mammography images." International Journal of Image, Graphics and Signal Processing 5.5 (2013): 47.

[2] Sreeja, G. Bharatha, P. Rathika, and D. Devaraj. "Detection of tumours in digital mammograms using wavelet based adaptive windowing method." International Journal of Modern Education and Computer Science 4.3 (2012): 57.

[3] Mina, Luqman Mahmood, and Nor Ashidi Mat Isa. "Preprocessing Technique for Mammographic Images."

[4] Wun, Lap-Ming, Ray M. Merrill, and Eric J. Feuer. "Estimating lifetime and age-conditional probabilities of developing cancer." Lifetime data analysis 4.2 (1998): 169-186.

[5] Gaikwad, Ms Nayan H., Ms Anjali P. Narwadkar, and Ms Ashwini S. Barge. "Enhancement of mammogram for detection of breast cancer using adaptive median filter." International Research Journal of Multidisciplinary Studies 2.3 (2016).

[6] Gonzalez, R. C., Woods R. E.: Digital Image Processing. 2nd edn. Prentice-Hall, NewJersey (2002).

[7] Chen, Soong-Der, and Abd Rahman Ramli. "Contrast enhancement using recursive mean-separate histogram equalization for scalable brightness preservation." IEEE Transactions on consumer Electronics 49.4 (2003): 13011309.

[8] Kim, Hyoung-Joon, et al. "Contrast enhancement using adaptively modified histogram equalization." PacificRim Symposium on Image and Video Technology. Springer Berlin Heidelberg, 2006. 
[9] Ibrahim, Haidi, and Nicholas Sia Pik Kong. "Brightness preserving dynamic histogram equalization for image contrast enhancement." IEEE Transactions on Consumer Electronics 53.4 (2007)

[10] Breast Cancer Facts \& Figures, 2013-2014, American Cancer Society, Inc.

[11] Ittannavar, S. S., and R. H. Havaldar. "Comparative Study of Mammogram Enhancement Techniques for Early Detection of Breast Cancer."

[12] Makandar, Aziz, and Bhagirathi Halalli. "Breast cancer image enhancement using median filter and clahe."
International Journal of Scientific \& Engineering Research 6.4 (2015): 462-465.

[13] Bandyopadhyay, Samir Kumar. "pre-processing of Mammogram Images." international journal of engineering science and technology 2.11 (2010): 67536758.

[14] Akila, K., L. S. Jayashree, and A. Vasuki. "Mammographic image enhancement using indirect contrast enhancement techniques-a comparative study." Procedia Computer Science 47 (2015): 255-261. 